\title{
Feature Extraction and Random Forest to Identify Sheep Behavior from Accelerometer Data
}

\author{
Natasa Kleanthous ${ }^{1}$, Abir Hussain ${ }^{1}$, Wasiq Khan ${ }^{1}$, Jenny Sneddon ${ }^{2}$, Alex Mason ${ }^{3}$ \\ Department of ${ }^{1}$ Computer Science, ${ }^{2}$ Natural Sciences and Psychology \\ Liverpool John Moores University, Liverpool UK \\ ${ }^{3}$ Faculty of Science and Technology, Norwegian University of Life Sciences, As, Norway \\ N.K.Orphanidou@2015.ljmu.ac.uk, A.Hussain@ljmu.ac.uk, \\ W.Khan@ljmu.ac.uk, J.C.Sneddon@ljmu.ac.uk, alex.mason@nmbu.no
}

\begin{abstract}
Sensor technologies play an essential part in the agricultural community and many other scientific and commercial communities. Accelerometer signals and Machine Learning techniques can be used to identify and observe behaviours of animals without the need for an exhaustive human observation which is labour intensive and time consuming. This study employed random forest algorithm to identify grazing, walking, scratching, and inactivity (standing, resting) of 8 Hebridean ewes located in Cheshire, Shotwick in the UK. We gathered accelerometer data from a sensor device which was fitted on the collar of the animals. The selection of the algorithm was based on previous research by which random forest achieved the best results among other benchmark techniques. Therefore, in this study, more focus was given to feature engineering to improve prediction performance. Seventeen features from time and frequency domain were calculated from the accelerometer measurements and the magnitude of the acceleration. Feature elimination was utilised in which highly correlated ones were removed, and only nine out of seventeen features were selected. The algorithm achieved an overall accuracy of $99.43 \%$ and a kappa value of $98.66 \%$. The accuracy for grazing, walking, scratching, and inactive was $99.08 \%, 99.13 \%$, $99.90 \%$, and $99.85 \%$, respectively. The overall results showed that there is a significant improvement over previous methods and studies for all mutually exclusive behaviours. Those results are promising, and the technique could be further tested for future real-time activity recognition.
\end{abstract}

Keywords: accelerometer data, animal activity recognition, feature extraction, machine learning, random forest , sheep behaviour ,signal processing

\section{$1 \quad$ Introduction}

Sheep play an essential role in our society as they are kept for meat, wool, as well as pasture management. According to research conducted, sheep are shown to be as effective as herbicides in controlling winter weed, as well as insecticides [1-2]. In order to manage the land they graze, human observation is the traditional mean of monitoring the distribution of the animals, which is a time consuming and labour intensive process. Thus, development of smart devices is essential for efficient monitoring and controlling of the animals' distribution on the pasture. 
Automated monitoring of animals also allows early detection of illness, particularly lameness; present in an estimated $80 \%$ of UK flocks [3] [4-6]. Furthermore, evidence showed that the reduced activity or decreased food intake of the animal might be an indicator of disease. Therefore, computerized monitoring of animals in real-time has become a pressing requirement in sheep production systems. Using insight from automatic monitoring capability can offer sufficient knowledge of the animal's welfare and food intake, and the decision making of the land and animal managers can be made more efficiently.

Accelerometers are widely used with machine learning techniques to identify animal behaviour such as cattle [7-14], horses [15], sharks [16], goats [17, 18] and other domesticated or wild animals. However, in this study, we focused only on previous research that involves sheep behaviour in order to identify challenges concerned with this type of animal and be able to compare between previous studies. Additionally, we aim to improve prediction performance of the activities of the animals.

The reminder of this paper is organized as follows. Section 2 consists of background information. Section 3 provides information about the materials and methodology, while Section 4 demonstrates results and discussions. Section 5 includes the conclusion and the future work.

\section{Background}

Marais et al. [19] developed a device capable of collecting accelerometer signals at $100 \mathrm{~Hz}$ from a collar. The authors extracted features using 5.12-second windows and applied linear discriminant analysis (LDA) and quadratic discriminant analysis (QDA) using 10 features to classify five common behaviours of the animals (lying, standing, walking, running and grazing). LDA and QDA achieved an overall accuracy of $87.1 \%$ and $89.7 \%$, respectively. Discriminant analysis was tested by Giovanetti et al. to classify grazing, ruminating, and resting of sheep using a 60 -second window and accelerometer data. The algorithm yielded an overall accuracy of $93.0 \%$ and $\mathrm{k}$ coefficient of $89.0 \%$ [20].

Nadimi et al. [21] classified five mutually exclusive behaviours (grazing, lying, walking, standing, and others) with $76.2 \%$ success rate. Additionally, they classified two behaviours (grazing and lying) with a success rate of $83.5 \%$ using the NguyenWidrow method and the Levenberg- Marquardt back-propagation algorithm. Compared to similar studies, the authors showed significant improvement of the designed system.

Kamminga et al. compared several machine learning algorithms to detect five mutually exclusive behaviours using data gathered from goats and sheep using accelerometer, gyroscope, and magnetometer signals. The best results were obtained using a $1 \mathrm{~s}$ window and Deep neural networks with a 94\% accuracy [22]. The same dataset were used by Kleanthous et al. that tested multilayer perceptron, random forests, extreme gradient boosting, and k-Nearest neighbors to classify sheep and goat behaviour [23]. The best results achieved using random forest algorithm and classified grazing, lying, scratching or biting, standing, and walking with an overall accuracy of $96.47 \%$ and 
kappa value of $95.41 \%$. The authors conducted another experiment and they gathered accelerometer and gyroscope data from more sheep using smartphones to test the performance of random forest and their previous method using a smaller sample rate; $10 \mathrm{~Hz}$ [24]. The technique proved successful and they achieved accuracy and kappa value of $96.43 \%$, and $95.02 \%$, respectively by using only accelerometer features.

Mansbridge et al. collected accelerometer and gyroscope signals from sensors attached to the ear and collar of sheep at $16 \mathrm{~Hz}$ [25]. Various machine learning algorithms were tested using multiple features from the signals. Random forest yielded the highest results using 39 feature characteristics and a $7 \mathrm{~s}$ window, achieving accuracy of $92 \%$ and $91 \%$ for collar and ear data, respectively.

Barwick et al. were also interested in applying machine learning to describe sheep behaviour using accelerometers to evaluate the effectiveness by placing accelerometers on different parts of the body; ear, collar, leg [26]. The authors applied QDA and best results obtained from the ear acceleration data, at $94 \%, 96 \%$ and $99 \%$ for grazing, standing, and walking, respectively.

Walton et al. evaluated sampling frequency $(8,16$, and $32 \mathrm{~Hz})$, window size $(3,5$, and $7 \mathrm{~s}$ ) and sensor position (ear and collar) to classify sheep behaviour using random forests [27]. Their results suggested that the $16 \mathrm{~Hz}$ sampling frequency and a 7 -second window offer benefits concerning battery energy and it has the potential to be used for real-time monitoring system. The authors achieved results of $91 \%-93 \%$ accuracy and F-score of $88 \%-95 \%$.

Alvarenga et al. [28] evaluated the performance of decision trees for accelerometer data obtained from sheep. The algorithm was validated for 3, 5 and 10-second epochs. The best results in terms of accuracy were achieved for the 5-second epoch having accuracy of $85.5 \%$.

The sheep activity was also evaluated from Le Roux et al. [29] The authors developed an energy-aware feature and classifier selection technique for low-power sensor applications to minimize the energy consumed and also minimizing the accuracy loss of the classifier. The sheep data that they used included accelerometer signals and the authors were able to achieve a reduction in energy consumption while achieving an accuracy of $88.4 \%$ for classification of five behaviours. The authors, in a previous study, also evaluated sheep behaviour based on accelerometer data [30]. The classification algorithm achieved an accuracy of $82.4 \%$ for standing, walking, grazing, running and lying behavioural classes.

Guo et al. gathered signals from an IMU (Inertial Measurement Unit) sensors at a $20 \mathrm{~Hz}$ sampling rate and compared the grazing behaviour results according to different sward surface heights [31]. The authors applied Linear discriminant analysis on several datasets which they consisted of three sward surface heights. Overall, they achieved accuracy over $95 \%$ with the best results achieved using a 10s window having accuracy of $98.2 \%$. The authors showed that the IMU sensors are capable of providing robust information on the grazing behaviour of the animal despite the sward surface heights.

Decandia et al. [32] evaluated the performance of canonical discriminant analysis (CDA), and discriminant analysis (DA) to distinguish between three behaviours of sheep; grazing, ruminating, and others. The authors aimed to identify the window which provides the best algorithm performance and they evaluated windows of 5, 10, 30, 60, 
120,180 and $300 \mathrm{~s}$ from accelerometer signals sampled at $62.5 \mathrm{~Hz}$. Best results were achieved with the 30s epoch having accuracy and kappa value of $89.7 \%$ and $80 \%$, respectively. Vazquez et al. [33] aimed to develop a combined online (k-means) and offline ( $\mathrm{k}$ nearest neighbors) algorithm, which deals with concept drift to deal with three behaviours of sheep. The combined algorithm produced results with average accuracies of $85.18 \%$, average specificities of $82.84 \%$, and an average recall of $57.82 \%$.

All of the abovementioned studies involve the use of Machine Learning techniques to identify sheep behaviour at pasture, however there is still a need for improvement of the prediction accuracy. The aim of our study is to significantly improve our previously tested method [24] by expanding the feature set and decreasing the sliding window to 5 seconds. In our experiment, we focused on four behaviours; grazing, walking, scratching, and inactive. For the experiment, we used only accelerometer data sampled at $12.5 \mathrm{~Hz}$, which was previously demonstrated adequate and did not compromise the battery life of the device [27].

\section{$3 \quad$ Materials and Methods}

This section describes the materials and methods used to examine the performance of Random Forest algorithm regarding the classification of four mutually exclusive behaviours of sheep; grazing, walking, scratching, and inactive. Fig. 1 , shows the process followed to conduct the study.



Fig. 1. Methodology

\subsection{Animals, Location, and sensor device}

This study was conducted in July-August 2019 in Cheshire Shotwick (OS location 333781,371970), UK. Eight Hebridean ewes between the ages of 5-12 years were fitted with a sensor device collar. The animals were free to use a paddock of $1500 \mathrm{~m}^{2}$ area size and had access to grass and water all the time. The Senior Research Officer and LSSU Manager of Liverpool John Moores University approved the protocol of the experiment (approval AH_NKO/2018-13). 
The MetamorionR ${ }^{\circledR}[34]$ wearable device was used for the current experiment. The sensor device collects motion and environmental data, however for this experiment we only used accelerometer measurements. The device weights $0.3 \mathrm{oz}$ and its dimensions are of $36 \mathrm{~mm} \times 27 \mathrm{~mm} \times 10 \mathrm{~mm}$ with the case. Additionally, a $60 \mathrm{mAH}$ MicroUSB rechargeable li-po battery powers it. For this study, we used only accelerometer measurements at a sample rate of $12.5 \mathrm{~Hz}$. The device logged and saved the data on its offboard memory as a CSV file.

\subsection{Data Collection and Annotation}

The animals were fitted with collars, which had the device attached in a nonfixed position to have a more generalised algorithm performance independent of the sensor orientation and position. The animals were video recorded during the morning, afternoon or night, and one observer was present each time. At the end of each day, the CSV file was saved for later use. Once all the recordings were completed with a total of 40 hours of recorded behaviours, the accelerometer readings were time synchronised with the video recordings for behavioural annotation. For animal behaviour annotation, we used ELAN_5.7_AVFX Freeware tool [35] and manually labeled the behaviours as grazing, walking, scratching, and inactive.

\subsection{Data Preprocessing}

After the data annotation, all the CSV files were merged and imported in Rstudio ${ }^{\circledR}$ for visualization and analysis. The behaviours of interest for this study were: grazing, walking, scratching, resting, and standing. Behaviours such as fighting, shaking, and rubbing were not considered for this study. This resulted in utilising 28 out of 40 hours for analysis. Missing values were present in the data and therefore they were eliminated. The behavioural data comprised of a set $\mathrm{A}=\left\{\mathrm{t}_{\mathrm{i}}, \mathrm{ax}_{\mathrm{i}}, \mathrm{ay}_{\mathrm{i}}, \mathrm{az}_{\mathrm{i}}, \mathrm{y}_{\mathrm{i}}\right\}$ for $\mathrm{i}=1, . ., \mathrm{n}$, where $\mathrm{n}$ is the number of observations. The $t$ is the timestamp, (ax, ay, az) are the accelerometer measurements, and $\mathrm{y}$ is the target vector where $\mathrm{y} \in$ \{grazing, walking, scratching, inactive\}. The following equations (1), (2), and (3) define the acceleration vector:

$$
\begin{gathered}
\text { ax }=1 \mathrm{~g} * \sin \theta \\
\text { ay }=-1 \mathrm{~g} * \sin \theta * \sin \phi \\
\mathrm{az}=1 \mathrm{~g} * \cos \theta
\end{gathered}
$$

Where $\theta$ is the angle between az relative to gravity, $\phi$ is the angle of ax relative to ground, and $\mathrm{g}$ is the gravitational constant where $1 \mathrm{~g}=9.81 \mathrm{~m} / \mathrm{s}^{2}$.In this step we extracted the magnitude of the acceleration (4):

$$
\text { Magnitude }=\sqrt{a x^{2}+a y^{2}+a z^{2}}
$$




\subsection{Feature Extraction, Feature Importance, and Dimensionality Reduction}

A total of 17 features were calculated from the $\mathrm{x}, \mathrm{y}, \mathrm{z}$, and magnitude of the acceleration signals for each activity resulting in a total of 68 newly created features (i.e. 17 features $\times 4$ activities). Those features include the mean, standard deviation, root mean square, root mean square velocity, energy, sum of changes, mean of changes, absolute and squared integrals, madogram [36], peak frequency, peak to peak value, kurtosis and skewness, zero crossing, crest factor, and signal entropy. The features were extracted using a 5 -second sliding window. Having a greater window in a real-time classification could provoke mislabeling because the animal might exhibit more than one behaviour in a short time interval; therefore, a 5-second window is considered sufficient.

The distributions for first four principal components (PCs) with respect to target class, original attributes and corresponding impacts of the target classes within the dataset are represented in Fig. 3(a) and (b). These figures also indicate the non-linearity of the problem specifically in terms of first four PCs covering the highest variances $(\sim 65 \%)$ within the overall principal components. Though, there is a small degree of overlap between all activities. However, this was expected since the head movements of the animal might exhibit similar patterns in some instances. Furthermore, the plots help to understand the corresponding influence of the features within the datasets on the classification of animal behaviours (i.e. 4 target classes). For instance, in Fig. 3(a) the madogram of the magnitude $\&$ the madogram of ay measurement have a clear impact on class 'inactive' as compared to root mean square velocity which influence the 'scratching and grazing' classes.

We used the most commonly used dimensionality reduction technique PCA[37] to identify the most significant attributes/features within the dataset set and eliminating the unnecessary features. In other words, PCA can be used to transform a large dataset containing large number of features/variables to a lower dimension which still holds most of the information contained in the original high dimensional dataset. One of the important properties of PCA is the attribute loadings on the principal components that can also be used for the identification of attribute importance within the original dataset. The correlation coefficient between the dataset attributes is represented by the principal components' loadings (i.e. obtained through PCA). The component rotations provide the maximized sum of variances of the squared loadings. The absolute sum of component rotations gives the degree of importance for the corresponding attributes in dataset. Fig. 4 shows the feature significance score within the original dataset which is calculated through the PCs loadings. There are variations in the importance measure of features which can be used to identify and hence remove the unnecessary features from 
the dataset. For instance, 'madogram' of $\mathrm{z}$, and $\mathrm{x}$ axis are indicated the top-ranked variables compared to magnitude 'integrals' and 'rms' of the ay axis which are indicated the least important variables within the original dataset.

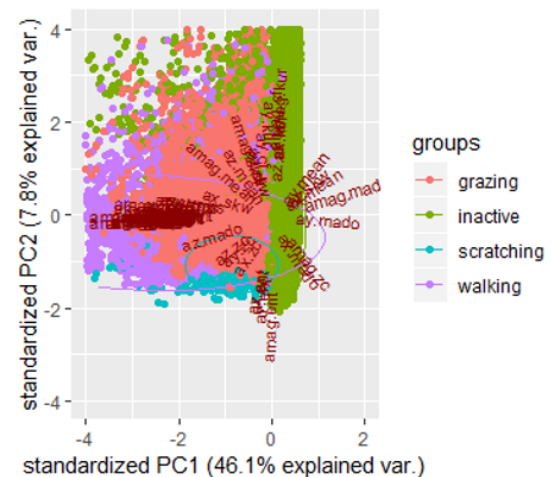

(a)

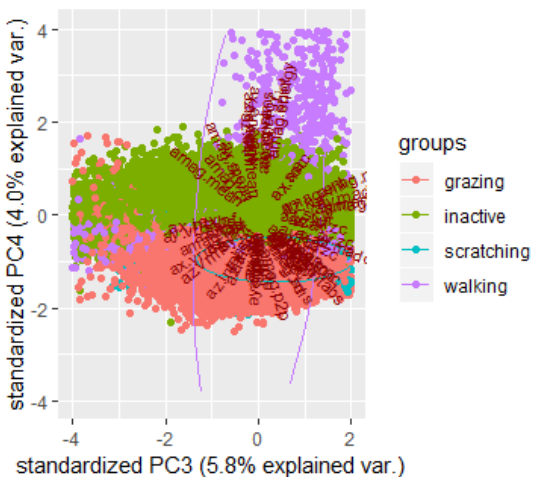

(b)

Fig. 2. (a) First two PCA components' distributions; (b) $3^{\text {rd }}$ and $4^{\text {th }}$ components' distributions within the PCA components

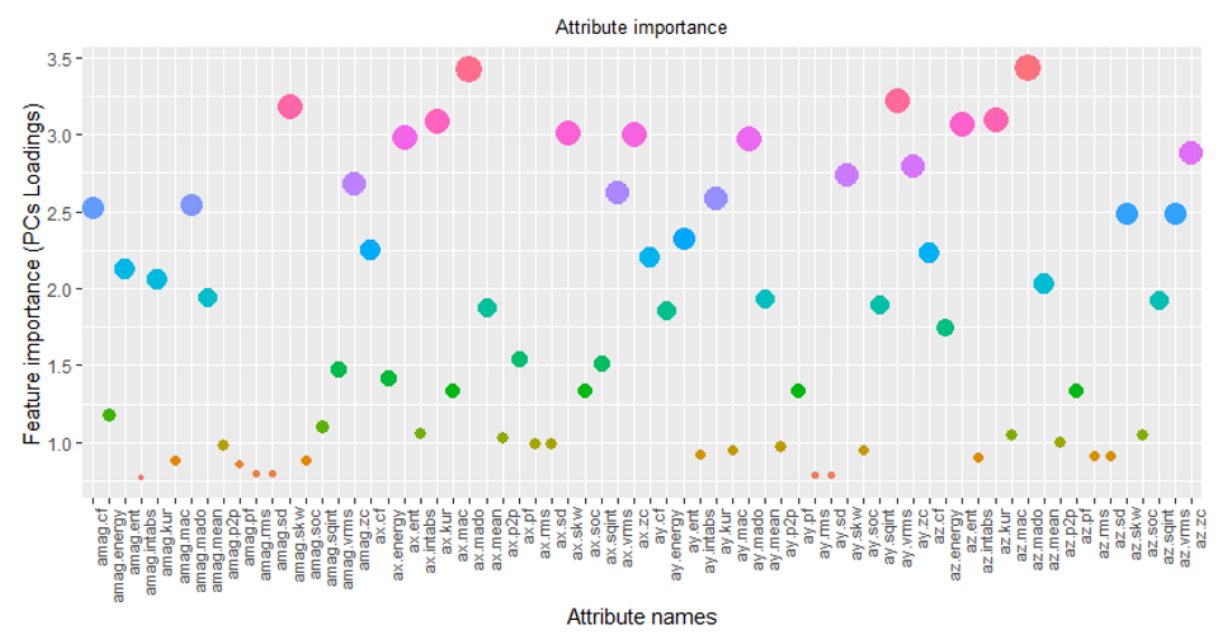

Fig. 3. Measure of feature importance within the Dataset using principal components loading

To further investigate the features/attributes within the dataset, we used the correlation coefficients. The correlated features with correlation above $80 \%$ were removed and the remaining features are in agreement with the feature importance ranking indicated by the PCA. Therefore, we eliminated our features from 17 to 9 . The remaining features are the mean, crest factor, root mean square velocity, skewness, kurtosis, madogram, zero crossing rate, squared integrals, and signal entropy. 


\subsection{Classification}

The classification algorithm selected to evaluate our dataset and test the activity prediction performance of the animals was the Random Forest as it was proved successful in our previous studies, as well as other studies concerned with animal behaviour [9$11,14,24]$. Random forest [38] is an ensemble method which consists of a combination of decision trees which are dependent on random values. All trees are sampled independently with the same distribution. The classification decision is then made based on the majority of votes from each tree.

To estimate the performance of the algorithm, we evaluated the model with the Out of bag (OOB) accuracy. The idea behind OOB was since the decision trees are learning from a subset of the dataset (63\%), then we have unseen data (37\%) to be used for evaluation. This method is a good estimate of the ability of the model to generalize on unseen data [39]. We then recursively evaluated the performance using sensitivity, specificity, accuracy, and kappa value quality measures by means of 10 -fold cross validation. The results are presented in the next section.

\section{$4 \quad$ Results and Discussion}

The performance of random forest is presented in Table 1. The four behaviours are classified correctly at a high rate. The overall accuracy of the algorithm is $99.43 \%$ with kappa value of $98.66 \%$. Additionally, the f1-score is between $91.53 \%-99.90 \%$. The lowest F1-score is resulted from scratching, and the highest from inactive behaviour. The sensitivities of all behaviours are between $98.26 \%$ to $99.87 \%$. Also, the specificities are between $99.60 \%$ to $99.92 \%$. Scratching was misclassified only once with grazing, while grazing was misclassified with scratching and walking in some cases. The same is valid with walking as it was misclassified with grazing and scratching.

Table 1. Random Forest Performance on unseen data

\begin{tabular}{|c|c|c|c|c|}
\hline \multirow{2}{*}{} & \multicolumn{4}{|c|}{ Activities } \\
\cline { 2 - 5 } & Grazing & Walking & Scratching & Inactive \\
\hline Sensitivity & $98.26 \%$ & $98.66 \%$ & $99.87 \%$ & $99.86 \%$ \\
\hline Specificity & $99.91 \%$ & $99.60 \%$ & $99.92 \%$ & $99.84 \%$ \\
\hline F1-score & $98.97 \%$ & $94.64 \%$ & $91.53 \%$ & $99.90 \%$ \\
\hline Balanced Accuracy & $99.08 \% \%$ & $99.13 \%$ & $99.90 \%$ & $99.85 \%$ \\
\hline \multicolumn{7}{|c|}{ Overall Accuracy : 99.43\%, Kohen's Kappa value $: \mathbf{9 8 . 6 6 \%}$} \\
\hline
\end{tabular}

Only limited cases misclassified inactive behaviour with the other behaviours However, the misclassification is limited, and consequently, the results showed high accuracy, sensitivity, and specificity in all 4 cases.

In this study, we noted that the movements the animals are conducting while they graze can sometimes have similarities with the walking and scratching behaviour. Additionally, resting and standing provide a similar pattern of the acceleration signals, because of the animals' inactive state and this was also noted by Barwick et al. [26]. On the other hand, while the animals scratch or bite, the activity is detected easily as 
the magnitude changes markedly. While the animals are ruminating, the head movements are relatively small, and stationary compared with grazing and it does not interfere with the correct classification of the activity they perform. From the results, we noted that 5 -second windows can provide a very good activity pattern representation and therefore could be suggested that this size is adequate. However, Decandia et al. [32], conducted experiments with various window sizes, such as 5, 10, 30, 60, 120, 180 and 300 seconds, and they identified that the best performance was obtained from a 30 second window having sensitivity $94.8 \%$ for grazing, $80.4 \%$ for ruminating, and $92.3 \%$ for other behaviours. Though, the two studies cannot be compared because the ML model applied, the selection of features, and the position of the sensor is different. On the other hand, a 5-second window achieved best performance in a study of Alvarenga et al.[28] when they compared 3, 5, and 10 second windows. The authors achieved an overall accuracy of $85.50 \%$ with Decision Trees and 5 second windows which exhibited higher accuracy in comparison with 3 and 10 second windows. However, the variety of feature combinations, ML techniques, sample rate, and window size used in previous and the current study show that there is still need for further investigation and there is no clear indication yet on the technique that is more suitable to be used for sheep activity recognition.

\section{Conclusion and Future Work}

This study was focused on detecting four mutually exclusive behaviours of interest to the animal health and production industry. Data was collected from eight Hebridean ewes located in Cheshire Shotwick, UK. Accelerometer signals were collected from a sensor which was attached on the collar of each animal. A total of 28 hours was used to test the performance of random forest to detect each behaviour. The behaviours of interest were the grazing, walking, scratching, and inactive. To test the algorithm, 17 features were extracted from the $\mathrm{x}, \mathrm{y}, \mathrm{z}$, and magnitude of the acceleration signal resulting in 68 newly created variables. We then removed features with higher than $80 \%$ correlation and eliminated the features to 9. The evaluation of the random forest algorithm was then assessed using out-of-bag (OBB) estimate which is empirically proven that is as accurate as using a test set of the same size as the training set [39].

The results were very high for all the activities having accuracies of $99.08 \%$ for grazing, $99.13 \%$ for walking, $99.90 \%$ for scratching, and $99.85 \%$ for inactive. The overall accuracy and kappa value were $99.43 \%$ and $98.66 \%$. The results showed that there is an important improvement over previous methods. The technique can be further tested and used for online activity recognition system and be part of a multifunctional smart device for monitoring and controlling animal behaviour and position. In future work, we will use GPS coordinates to track the position of the animals and monitor the land they mostly graze. The implementation of such a device can be used as an intelligent assistant to provide valuable information regarding the food intake of the animals and their activities during the day, which can improve the decision making of the land managers. Such information can contribute to the animal's welfare, pasture utilisation and overall farm and animal decision management approach. 


\section{Acknowledgment}

We would like to thank the Douglas Bomford Trust [40] for the funding support of this study

\section{References}

1. Umberger, S.H.: Sheep Grazing Management.

2. Doran, M.P., Hazeltine, L., Long, R.F., Putnam, D.H.: Strategic Grazing of Alfalfa by Sheep in California's Central Valley. (2010).

3. Winter, A.C.: Lameness in sheep. Small Ruminant Research. 76, 149-153 (2008).

4. Barwick, J., Lamb, D., Dobos, R., Schneider, D., Welch, M., Trotter, M.: Predicting lameness in sheep activity using tri-axial acceleration signals. Animals. 8, 1-16 (2018).

5. Al-Rubaye, Z., Al-Sherbaz, A., McCormick, W.D., Turner, S.J.: The use of multivariable wireless sensor data to early detect lameness in sheep. (2016).

6. Gougoulis, D.A., Kyriazakis, I., Fthenakis, G.C.: Diagnostic significance of behaviour changes of sheep: A selected review. Small Ruminant Research. 92, 52-56 (2010).

7. González, L.A.A., Bishop-Hurley, G.J.J., Handcock, R.N.N., Crossman, C.: Behavioral classification of data from collars containing motion sensors in grazing cattle. Computers and Electronics in Agriculture. 110, 91-102 (2015).

8. Robert, B., White, B.J., Renter, D.G., Larson, R.L.: Evaluation of three-dimensional accelerometers to monitor and classify behavior patterns in cattle. Computers and Electronics in Agriculture. 67, 80-84 (2009).

9. Rahman, A., Smith, D. V., Little, B., Ingham, A.B., Greenwood, P.L., Bishop-Hurley, G.J.: Cattle behaviour classification from collar, halter, and ear tag sensors. Information Processing in Agriculture. (2018).

10. Smith, D., Rahman, A., Bishop-Hurley, G.J., Hills, J., Shahriar, S., Henry, D., Rawnsley, R.: Behavior classification of cows fitted with motion collars: Decomposing multi-class classification into a set of binary problems. Computers and Electronics in Agriculture. 131, 40-50 (2016).

11. Dutta, R., Smith, D., Rawnsley, R., Bishop-Hurley, G., Hills, J., Timms, G., Henry, D.: Dynamic cattle behavioural classification using supervised ensemble classifiers. Computers and Electronics in Agriculture. 111, 18-28 (2015).

12. Andriamandroso, A.L.H., Lebeau, F., Beckers, Y., Froidmont, E., Dufrasne, I., Heinesch, B., Dumortier, P., Blanchy, G., Blaise, Y., Bindelle, J.: Development of an open-source algorithm based on inertial measurement units (IMU) of a smartphone to detect cattle grass intake and ruminating behaviors. Computers and Electronics in Agriculture. 139, 126-137 (2017).

13. Riaboff, L., Aubin, S., Bédère, N., Couvreur, S., Madouasse, A., Goumand, E., Chauvin, A., Plantier, G.: Evaluation of pre-processing methods for the prediction of cattle behaviour from accelerometer data. Computers and Electronics in Agriculture. 165, 104961 (2019).

14. Vázquez Diosdado, J.A., Barker, Z.E., Hodges, H.R., Amory, J.R., Croft, D.P., Bell, N.J., Codling, E.A.: Classification of behaviour in housed dairy cows using an accelerometer-based activity monitoring system. Animal Biotelemetry. 3, (2015). 
15. Gutierrez-Galan, D., Dominguez-Morales, J.P., Cerezuela-Escudero, E., Rios-Navarro, A., Tapiador-Morales, R., Rivas-Perez, M., Dominguez-Morales, M., JimenezFernandez, A., Linares-Barranco, A.: Embedded neural network for real-time animal behavior classification. Neurocomputing. 272, 17-26 (2018).

16. Hounslow, J.L.L., Brewster, L.R.R., Lear, K.O.O., Guttridge, T.L.L., Daly, R., Whitney, N.M.M., Gleiss, A.C.C.: Assessing the effects of sampling frequency on behavioural classification of accelerometer data. Journal of Experimental Marine Biology and Ecology. 512, 22-30 (2019).

17. Navon, S., Mizrach, A., Hetzroni, A., Ungar, E.D.: Automatic recognition of jaw movements in free-ranging cattle, goats and sheep, using acoustic monitoring. Biosystems Engineering. 114, 474-483 (2013).

18. Kamminga, J.W., Le, D. V., Meijers, J.P., Bisby, H., Meratnia, N., Havinga, P.J.M.: Robust Sensor-Orientation-Independent Feature Selection for Animal Activity Recognition on Collar Tags. Proceedings of the ACM on Interactive, Mobile, Wearable and Ubiquitous Technologies. 2, 1-27 (2018).

19. Marais, J., Wolhuter, R., Niesler, T., Le Roux, S., Wolhuter, R., Niesler, T., Le Roux, S., Petrus, S., Roux, L., Wolhuter, R., Niesler, T.: Automatic classification of sheep behaviour using 3-axis accelerometer data. (2014).

20. Giovanetti, V., Decandia, M., Molle, G., Acciaro, M., Mameli, M., Cabiddu, A., Cossu, R., Serra, M.G., Manca, C., Rassu, S.P.G.G., Dimauro, C.: Automatic classification system for grazing, ruminating and resting behaviour of dairy sheep using a tri-axial accelerometer. Livestock Science. 196, 42-48 (2017).

21. Nadimi, E.S., Jørgensen, R.N., Blanes-Vidal, V., Christensen, S.: Monitoring and classifying animal behavior using ZigBee-based mobile ad hoc wireless sensor networks and artificial neural networks. Computers and Electronics in Agriculture. 82, 44-54 (2012).

22. Kamminga, J.W., Bisby, H.C., Le, D. V., Meratnia, N., Havinga, P.J.M.: Generic Online Animal Activity Recognition on Collar Tags. In: Proceedings of the 2017 ACM International Joint Conference on Pervasive and Ubiquitous Computing and Proceedings of the 2017 ACM International Symposium on Wearable Computers on - UbiComp '17. pp. 597-606. ACM, New York, NY, USA (2017).

23. Kleanthous, N., Hussain, A., Mason, A., Sneddon, J., Shaw, A., Fergus, P., Chalmers, C., Al-Jumeily, D.: Machine learning techniques for classification of livestock behavior. In: Lecture Notes in Computer Science (including subseries Lecture Notes in Artificial Intelligence and Lecture Notes in Bioinformatics). pp. 304-315 (2018).

24. Kleanthous, N., Hussain, A., Mason, A., Sneddon, J.: Data Science Approaches for the Analysis of Animal Behaviours. In: Lecture Notes in Computer Science (including subseries Lecture Notes in Artificial Intelligence and Lecture Notes in Bioinformatics). pp. 411-422 (2019).

25. Mansbridge, N., Mitsch, J., Bollard, N., Ellis, K., Miguel-Pacheco, G.G., Dottorini, T., Kaler, J.: Feature selection and comparison of machine learning algorithms in classification of grazing and rumination behaviour in sheep. Sensors (Switzerland). 18, $1-16$ (2018).

26. Barwick, J., Lamb, D.W., Dobos, R., Welch, M., Trotter, M.: Categorising sheep activity using a tri-axial accelerometer. Computers and Electronics in Agriculture. 145, 289-297 (2018). 
27. Walton, E., Casey, C., Mitsch, J., Vázquez-Diosdado, J.A., Yan, J., Dottorini, T., Ellis, K.A., Winterlich, A., Kaler, J.: Evaluation of sampling frequency, window size and sensor position for classification of sheep behaviour. Royal Society Open Science. 5, (2018).

28. Alvarenga, F.A.P., Borges, I., Palkovič, L., Rodina, J., Oddy, V.H., Dobos, R.C.: Using a three-axis accelerometer to identify and classify sheep behaviour at pasture. Applied Animal Behaviour Science. 181, 91-99 (2016).

29. le Roux, S.P., Wolhuter, R., Niesler, T.: Energy-Aware Feature and Model Selection for Onboard Behavior Classification in Low-Power Animal Borne Sensor Applications. IEEE Sensors Journal. 19, 2722-2734 (2019).

30. Le Roux, S., Wolhuter, R., Niesler, T., Roux, S., Wolhuter, R., Niesler, T., Le Roux, S., Wolhuter, R., Niesler, T.: An Overview of Automatic Behaviour Classification for Animal-Borne Sensor Applications in South Africa. (2017).

31. Guo, L., Welch, M., Dobos, R., Kwan, P., Wang, W.: Comparison of grazing behaviour of sheep on pasture with different sward surface heights using an inertial measurement unit sensor. Computers and Electronics in Agriculture. 150, 394-401 (2018).

32. Decandia, M., Giovanetti, V., Molle, G., Acciaro, M., Mameli, M., Cabiddu, A., Cossu, R., Serra, M.G., Manca, C., Rassu, S.P.G., Dimauro, C.: The effect of different time epoch settings on the classification of sheep behaviour using tri-axial accelerometry. Computers and Electronics in Agriculture. 154, 112-119 (2018).

33. Vázquez-Diosdado, J.A., Paul, V., Ellis, K.A., Coates, D., Loomba, R., Kaler, J.: A combined offline and online algorithm for real-time and long-term classification of sheep behaviour: Novel approach for precision livestock farming. Sensors (Switzerland). 19, (2019).

34. MBIENTLAB INC: MetaMotionR - MbientLab, https://mbientlab.com/metamotionr/.

35. ELAN - The Language Archive, https://tla.mpi.nl/tools/tla-tools/elan/.

36. Gneiting, T., Ševčíková, H., Percival, D.B.: Estimators of fractal dimension: Assessing the roughness of time series and spatial data. Statistical Science. 247-277 (2012).

37. Wold, S., Esbensen, K., Geladi, P.: Principal component analysis. Chemometrics and Intelligent Laboratory Systems. 2, 37-52 (1987).

38. Breiman, L.: Random Forests. Machine Learning. 45, 5-32 (2001).

39. Breiman, L.: out-of-bag estimation, Technical Report. 1-13 (1996).

40. The Douglas Bomford Trust, https://www.dbt.org.uk/. 